4. Rümk. 1172

5. Rümk. 1317

6. B. Z. 521

7. Rümk. 1347

8. B. Z. 395

B. Z. 521

Angenommen

9. B.Z. 521

10. Berl. A. N. 60.189

11. B.D. +25 878 vergl. mit a $52837.86+253011.3$

12. Arg. Mer. +250929

a. B. Z. 523
$53434.50+252831.5$

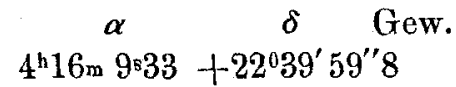

$44831.31+242311.7$

$4518.51+241752.7$

$45351.25+242733.3$

$45857.30+242640.21$

57.04

35.31

$45857.17+242637.7$

$51853.25+251012.3$

$52238.10+251351.7$

$52725.61+254427.2$
1. Altona A. N. 45.187

Antigone. $\alpha \quad \delta \quad$ Gew.

$846^{m} 59^{9} 58+20^{\circ} 3^{\prime} 16^{\prime \prime} 5$

2. Arg. Mer. +2002243 $84924.80+20201.1$

3. B. Z: 277

$84940.58+204112.2$

4. B.D. $+20^{\circ} 2249$ vergl.mit3 $85124.91+204037.9$

5. Weisse $8^{\text {h }} 1314$ vergl. mit a $85441.47+204953.2$

6. Lal. 17934

B. Z. 275

Angenommen

$\alpha^{\prime}-\alpha$ und $\delta^{\prime}-\delta$ sind wegen Refraction corrigirt und die mittleren Oerter der Sterne in A. R. auf Wolfers, in Decl. auf Auwers bezogen. Als Beobachter bezeichnet $\mathrm{M}=$ Möller, $\mathrm{D}=$ Dunèr und $\mathrm{L}=$ Lindstedt. Lund 1873, Mai 12.

Axel Möller.

\title{
Schreiben des Herrn E. Stephan, Directors der Sternwarte in Marseille, an den Herausgeber.
}

Marseille, le 1. Juin 1872.

La planète (131) a été trouvée par M. Borelly, d'après les indications de la dépèche américaine, le jour mème où celle-ci nous est parvenue. Je m'empresse de vous communiquer nos quatre premières observations:

Date. Heure de l'observ.

1873 (T.m.d.l'observ.) All d. (131)

Mai $27 \quad 11 \mathrm{~h} 21 \mathrm{~m} 15^{\mathrm{s}} \quad 16 \mathrm{~h} 13 \mathrm{~m} 27 \mathrm{~s} .29$

$\begin{array}{lllllll}28 & 11 & 46 & 59 & 16 & 12 & 24.29\end{array}$

1. f. p. P. d. (131)

$2.783 \quad 111^{0^{\prime}} 18^{\prime} 13^{\prime \prime} 0$

1. f. $\mathrm{p}$

*d. Comp.

Obs.

$\begin{array}{lllllll}29 & 11 & 4 & 47 & 16 & 11 & 23.85\end{array}$

p. nulle $\quad \begin{array}{lll}111 & 18 & 31.9\end{array}$

0.905 .2

a

Borelly

$\begin{array}{llll}2.815 & 111 & 18 & 36.3\end{array}$

0.906 .4

Coggia

$\begin{array}{lll}16 & 10 & 19.27\end{array}$

2.728

$\begin{array}{lll}111 & 18 & 41.2\end{array}$

0.904 .6

Stephan

0.905 .5

b

Coggia

Positions moy. des étoiles de comparaison pour 1873.0:

\begin{tabular}{|c|c|c|c|c|}
\hline * d. Comp. & & $\begin{array}{c}\mathrm{AR} \\
16 \mathrm{~h} 13 \mathrm{~m} 12 \mathrm{~s} .38\end{array}$ & $\begin{array}{l}\mathrm{P} \\
1111^{\prime} 31^{\prime} \quad 51^{\prime \prime 9} 9\end{array}$ & $\begin{array}{l}\text { Autorité } \\
\text { apportée à } 29547\end{array}$ \\
\hline$b$ & $8 e$ & $\begin{array}{lll}16 & 14 & 39.79\end{array}$ & $\begin{array}{lll}111 & 20 & 55.3\end{array}$ & id. \\
\hline
\end{tabular}

La première observation a été faite avec le chercheur de comètes dont la stabilité est très mediocre et par un fort mouvaís temps; les trois dernières ont été faites avec un excellent équatorial d'Eichens.

$\mathrm{J}_{\mathrm{e}}$ vous adresse également une nonvelle observation de la \& II 1867:

Dat. Heure de l'observ.

1873 (t. m. d. l'observ.)

Mai 29 $11 \mathrm{~h} 49 \mathrm{~m} 56 \mathrm{~s}$

AR d. $\not 4$ d. Com. I. f. p.

P d. \& $16^{\mathrm{h}} 25^{\mathrm{m} 4} 4 \mathrm{~s} .64 \quad 3.94$ $107^{\circ} 25^{\prime} 16^{\prime \prime} 5$

1. f. p.

Obs. Pos. m. de l'étoile de comp. 30030 Lal. oph. $8 \mathrm{e} \quad 16 \mathrm{~h} 24 \mathrm{~m} 18 \mathrm{~s} .74$

$\mathbf{P}$ $107^{\circ} 26^{\prime \prime} 7^{\prime \prime} 2$

Cette observation concorde assez bien avec la dernière éphéméride publiée par M. $d$ Asten.

E. Stephan.

Beobachtungen von Sonnenflecken, angestellt auf der Leipziger Sternwarte im Jahre 1872. von Herrn H. Leppig. Mitgetheilt von Herrn Prof. Dr, Bruhns.

Die nachstehend gegebenen Beobachtungen enthalten die Zählung der Gruppen und Flecken, sowie

Bemerkungen über gesehene Fackeln und über die Form der Penumbra bei Flecken am Sonnenrande. 
Die Sonne wurde im Jahre 1872 beobachtet an 198 Tagen und an keinem dieser Tage ohne Flecken gesehen.

Das zu den Beobachtungen angewandte Fernrohr ist ein 4 füssiger Frauenhofer, die Vergrösserung eine 80 fache.

Die notirte Zeit ist mittlere Leipziger. Januar.

29. $9 \mathrm{~h} 8 \mathrm{Gr}$. mit $25 \mathrm{Fl}$.

1. $12 \mathrm{~h} 2$ Grupp. mit $7 \mathrm{Fl}$.

2. $126, " 20$,

4. $126,, 21$,

6. $125, ", 16$,

8. $127, " 15$,

12. $127,, 21$,

18. 105,21,

19. $124,, 21$,

$22.125, ", 21$,

26. $124 \%, 15$ "

29. 46 ", "21,

31. 45 " "30",

Februar.

1. $12 \mathrm{~h} 8 \mathrm{Gr}$. mit $33 \mathrm{Fl}$.

2. 129,38 .

3. $129 " 38$,

$9.128, \% 21 \%$

10. 1210,22,

11. $128,, 30 \%$

14. $126 \% 19$,

18. $124 \% 11 \%$

22. 46 " 22 ,"

27. $127,, 27$,

29. $129 \% 30 \%$

März.

3. $12 \mathrm{~h} 12$ Gr. mit $26 \mathrm{Fl}$.

4. 127,14 ,

5. $47 \% 17$,

6. $44 \% 10 \%$

7. 43

8. 124

9. $12 \quad 5$

12. 4.2

13. 42

14. 41

15. $4 \quad 2$

16. 122

21. $12 \quad 10$

22. $4 \quad 13$

26. 46

27. 127

30. $4 \quad 11$ April.

2. $3^{\text {h }} 9$ Gr. mit 26 Fl.

3. 46

7. 12

11. 44 .

12. 4

13. 5

17. 5

18. 4

20. 10

22. 4

23. 4

25. 4

26. 5

27. 10

28. 9

30. 4

Mai.

1. $4^{\text {h }} 6$ Gr. mit 19 Fl.

2. 46

3. 45

7. 4

8. 57 ,

14. $97 .$,

15. 46 ,

16. $46 \%$

17. $4 \quad 4$,

18. $10 \quad 3$

23. $5 \quad 10$

6,

10 ,

13,

3,

2 ,

2,

3 ,

24. $4 \quad 6$

26. 87

27. 55

29. $9 \quad 11$

30. 911

31. $4 \quad 10$

Juni.

32,

27,

29,

29 ,"

20 ,

23,

16 ,

22,

55,

2 ,

1. $10 \mathrm{~h} 9 \mathrm{Gr}$. mit $26 \mathrm{Fl}$.

22,

30 ,"

22,

3. 410

4. 116

5. 10

16,

, 16,

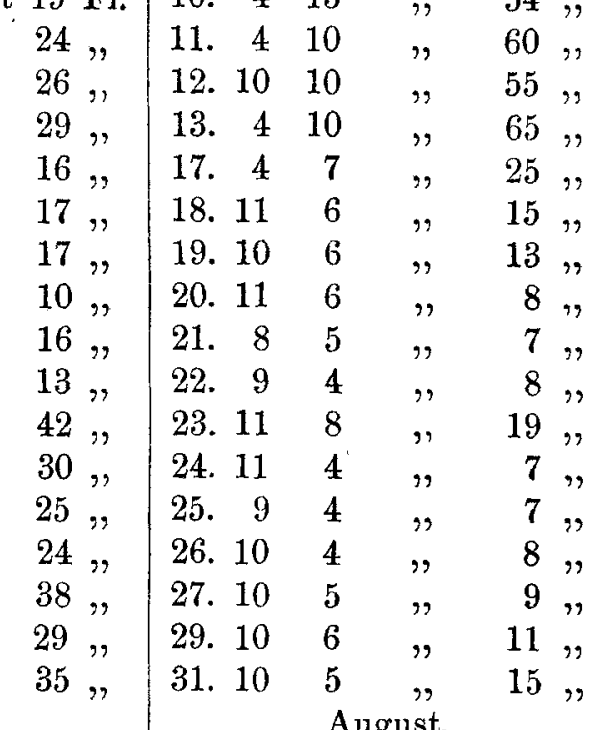

7. $4^{\text {h }} 5$ Gr. mit 15 Fl

8. 46,14,

10. $4 \quad 10 \quad, \quad 35 \%$

11. $108 \% 31$,

12. $48 \quad, \quad 40$,

14. $114 " 27$,

15. $114 \Longrightarrow 20$

17. 113 " 16 ,

19. $116 " 11$ "

20. $117 " 17$,

21. $118 \% 27$,

22. $56 \quad \%, 15$,

23. $106 \% 14$,

24. $115 \% 7$,

25. 107 , 18 ,

27. $49, \quad 27$,

29. 47 " 36 ,

1. $10 \mathrm{~h} 4 \mathrm{Gr}$. mit 14 Fl.

2. 56 " 25 ,

3. $45 \% 15$,

4. 45,217 ,

5. 47 , 26,

6. $48 \% 18$,

7. $118, " 30$,

8. $108 \quad, 23$,

9. 10.12

10. $4 \quad 13$

11. 410

12. $10 \quad 10$

13. $4 \quad 10$

18. 11

9. 10
1. $9 \mathrm{~h} 5$ Gr. mit $15 \mathrm{Gr}$

2. 46 " 17 ,

5. 44 " 12 ",

6. $98,36 "$

7. $97 \% 35 \%$
8. 5h 8 Gr. mit 32 Fl.

9. 57,30 ,

10. $108 \% 30$ "

12. $107 \% 18$,

13. $56{ }^{\circ} " 12$ "

14. $94 \%$ " 14 "

16. 56 " 18 ,

17. 106 , 15 ,

19. 34 " 44 "

20. 57 " 20 "

23. $114 \% 26$ "

24. $106 \% 20 \%$

25. $105 \% 13$,

26. $1010 \% 13 \%$

28. $104 \% 10$,

30. $1010 \% 9$ "

September.

1. $11 \mathrm{~h} 4 \mathrm{Gr}$. mit $12 \mathrm{Fl}$.

2. 115

8. 128 \% 26 ,

9. $107 \% 20$ \%

10. 49 " 34 ,

12. $95 \% 18$ "

14. 46 " 16 ,

16. $95 \% 13$ \%

17. $45 \% 11$,

19. $116 \% 14$ \%

20. $117 \% 15$,

21. $\begin{array}{lll}15 & 6 & \end{array}$

23. 97 7 24 ,

24. $11 \quad 10 \% 17$ "

29. $911 \% 40$,

October.

1. $10 \mathrm{~h} 11 \mathrm{Gr}$. mit $40 \mathrm{Fl}$.

3. 911

$\pi-41$,

$\begin{array}{llll}9 & 8 & 13\end{array}$

8. $94 \quad \% 7$ "

11. $85 \% 11$,

13. $116 \% 11$,

17. $106 \% 24$,

18. $127 \% 20 \%$

20. $105 \% 12$,

22. $127 \% 15$ \%

23. $44 \Rightarrow 10$ " 
26. $11 \mathrm{~h} 8 \mathrm{Gr}$ mit $30 \mathrm{Fl}$.

27. $119 \% 27$ \%

November.

1. $12^{\mathrm{h}} 6 \mathrm{Gr}$. mit $18 \mathrm{Fl}$.

3. $116 \% 9$,

8. $126 \% 23$ "

9. $127 \% 28$ "

10. $27 \% 26$ "

14. $106 \% 18$ "

17. $115 \% 28$ "

18. $128 \% 37 "$

21. 124 " 28 "

23. $124 " 30$ "

25. 114 " 25 "

Bemerkungen.

Fackeln. Januar 1., 2., 8., 12., 18., 19., 22., 26., 29. - Februar 1., 2., 3., 9., 10., 11., 14., 22., 27., 29. März 3., 4., 5., 6., 7., 8., 9., 12., 14., 15., 22., 26., 27., 29., 30. - April 2., 3, 7., 12., 13., 17., 18., 20., 22., 23., 25., 26., 27., 28., 30. - Mai 1., 2., 3., 7., 8., 14., 15., 16., 17., 18., 23., 24., 26., 27., 29., 30., 31. Juni 1., 3., 4., 5., 6., 7., 8., 10., 11., 12., 20., 21., 22., 23., 25., 27., 29. - Juli 3., 4., 5., 6., 7., 8., 9., 10., 13., 17., 18., 19., 20., 21., 22., 23., 24., 25., 27., 29., 31. - August 1., 2., 6., 7., 8., 9., 10., 12., 13., 14.,
16., 17., 20., 23. 24., 26., 28., 30. - September 1., 2., 5., 6., 7. 8., 9., 10., 12., 14., 16., 17., 20., 21., 23., 24., 27. - October 1., 3., 7., 8., 17., 18., 20., 22., 26., 27. - November 1., 3., 8., 9., 10., 14., 17., 18., 21., 23., 25. - Derember 1., 6., 8., 25., 26., 27., 29.

Die Penumbra wurde bei Flecken in der Nähe des Sonnenrandes nach dem Sonnenrande zu breiter gesehen: Februar 22. (bei 2 Flecken); April 22. (O. R.), blieb so bis zum 28.; Juli 3. (W. R.), 10. (W. R.); August 9. (O. R.), 30. (O. R.); September 6. (W. R.), 7. (W R.), 14. (O. R.); October 26. (O. R.); November 18. (O. R.).

Die Penumbra wurde bei einem Flecken am 3. Juni nahe dem W. R. nach dem W.R. zu schmäler geseben.

December 26. 12h lebhafte Lichtentwickelung an einer Stelle des O. R., an dieser Stelle December 27. $12 \mathrm{~h}$ grössere Gruppen von Flecken.

Bedeckung $\sigma$ Sagittarii vom Monde August 15. 1872 beobachtet auf der Leipziger Sternwarte von $\boldsymbol{H}$. Leppiq. б Sagittarii Eintritt $18 \mathrm{~h} 37 \mathrm{~m} 25 \mathrm{~s} .3$ Sternzeit (gut) Austritt $19 \quad 40 \quad 22.1 \quad$ (etwasuns.) 4 füssiger Fraunhofer, 80 fache Vergrösserung.

Leipzig, den 10. März 1873.

\section{Bruhns.}

\section{Anzeige.}

Es ist schon in den früheren Bänden bemerkt worden, dass ohne ausdrückliche Bestellung und Vorausbezahlung keine Nummer eines neuen Bandes versandt wird. Die Herren Abonnenten, welche diese Blätter fortzusetzen wünschen, werden also ersucht, um Unterbrechungen zu vermeiden, baldmöglichst ihre Bestellungen einzusenden.

Man pränumerirt bei der Expedition dieses Blattes (Kiel, Königl. Sternwarte) mit 3 Thlr. 6 Sgr. Preussisch Conrant und von diesem Preise wird auch den Buchhandlungen und Postämtern kein Rabatt gegeben, die also nothwendig ihren Abnehmern höhere Preise berechnen müssen. - Ueberbaupt sind alle in dieser Anzeige bemerkten Preise Nettopreise.

Für die mit der Post versandten Exemplare findet eine kleine Erhöhung statt, so dass der Preis für den Band, incl. Porto, sich stellt: für Deutschland und Oesterreich auf 4 Thlr. Preuss. Cour., für England auf 15 sb., für Frankreich und Italien auf $17^{1 / 4}$ Frcs., für Nordamerika auf $3^{2} / 3$ Dollars, für Holland auf $11 / 2$ Holl. Ducaten.

Einzelne Nummern werden nur zur Completirung, wenn sie vorräthig sind, à 5 Sgr. abgelassen.

\section{In halt:}

(Zu No. 1944): Schreiben des Herrn Hind, Superintendenten des Nautical Almanac an den Herausgeber. 369. - Beobachtungen kleiner Planelen auf der Sternwarte in Lund. Von Hern Prof. Axel Mibller. 369. - Schreiben des Herrn E. Stephan, Directors der Sternwarte in Marseille, an den Herausgeber. 379. - Beobachtungen von Sonnenflecken, angestellt auf der Leipziger Sternwarte im Jahre 1872 von Hern H. Leppig. Mitgetheilt von Herrn Prof. Dr. Bruhns. 379. - Anzeige. 383. 